\title{
Management of Live Cervical Ectopic Pregnancy
}

\author{
Nnadozie Igbokwe*, Dushyanthy Jeyanesan and Anku Mehta
}

Department of Obstetrics and Gynecology, Watford General Hospital, UK

*Corresponding author: Dr. Nnadozie Igbokwe, Specialist Registrar in Obstetrics and Gynecology, Watford General Hospital, UK; Tel: 01923244 366; Email: dozzybarry4@gmail.com

Received: February 17, 2020; Accepted: March 6, 2020; Published: March 13, 2020

\section{Abstract}

We report a case of live cervical ectopic pregnancy (CEP) at 6 weeks gestation. A 36-year-old nulliparous who presented with mild vaginal bleeding. She was haemodynamically stable with a serum B-hCG of 10713. A transvaginal ultrasound scan showed an empty uterus and lives cervical ectopic pregnancy with a fetal pole measuring $7.1 \mathrm{~mm}$ and yolk sac with negative sliding sign. She was counselled on options of management and had hysteroscopy and surgical evacuation under ultrasound guidance with no complications and post-operative methotrexate injection. She had a significant drop in her serial B-hCG and urine pregnancy test 3 weeks after surgery was negative.

Keywords: Cervical ectopic; Methotrexate; B-hCG; Pelvic ultrasound; Gestational sac; Fetal pole; Hysteroscopy

\section{Background}

Successful management of CEP is dependent on early and accurate diagnosis [1]. In view of the massive bleeding that is associated with surgical management, there is need for preoperative Multidisciplinary Team (MDT) approach involving experienced gynaecologists, interventional radiologists, haematologists, blood transfusion services etc. [2].

CEP is rare and it accounts for less than $1 \%$ of all ectopic pregnancies. It an ultrasound diagnosis and the following criteria may be used for accurate diagnosis:

- Uterine cavity visualised to be empty

- Barrel-shaped cervix

- No 'sliding-sign'

- Gestational sac seen below the level of the internal ostium of the cervix

- Vascularity around the gestational sac seen with colour Doppler [3].

We report a case of live CEP with high serum B-hCG of over $10,000 \mathrm{U} / \mathrm{L}$, in a 36 year old stable patient who underwent a successful hysteroscopy and suction curettage with no massive haemorrhage, and later had post-operative methotrexate injection. The sonographic findings in our patient were consistent with the above criteria, and her management was MDT based.

\section{Case Presentation}

A 36-year-old G1P0+0, with 6 weeks pregnancy presented with one day history of mild bleeding from the vagina with no abdominal pain. No history of nausea, vomiting, constipation or diarrhoea.

Her period was regular running 5 days in 28-30 days cycle, and no significant past gynaecological history. She had no previous history of pelvic inflammatory disease and her cervical screening was up to date and normal. There was no history of use of contraception she had no significant past medical or surgical history. She was married with no history of smoking and had no drug allergy.

On examination, she was stable with blood pressure of $131 / 73 \mathrm{mmHg}$ and pulse rate 80 beats per minute. Abdominal examination was soft, non-tender with no pelvic masses felt. Speculum examination showed no active bleeding. The Cervix was healthy looking and closed, and bimanual examination of the adnexae was unremarkable.

An assessment of Threatened miscarriage was made to rule out ectopic pregnancy. Patient was given information leaflets and booked for urgent pelvic ultrasound.

\section{Investigations}

Blood tests showed serum B-hCG of $10713 \mathrm{U} / \mathrm{L}$, with progesterone level of $44 \mathrm{mg} / \mathrm{ml}$. Urinalysis only showed 3+ of erythrocytes and Haemoglobin (HB) level was 149 g/L. Group and save was also done as per Trust guideline.

Transvaginal pelvic ultrasound showed anteverted uterus, normal size and appearance with heterogenous endometrial thickness of $12 \mathrm{~mm}$. There was a gestational sac with mean diameter of $9.7 \mathrm{~mm}$ located within the cervical canal with good decidual reaction and demonstrated large amount of vascularity with negative sliding sign. Within the gestational sac, a yolk sac was visible, with foetal pole measuring $7.1 \mathrm{~mm}$ equivalent to 6 weeks and 4 days pregnancy with cardiac pulsation. Ovaries were normal with no adnexal masses and no fluid in the pelvis. Left ovary measured $25 \times 11 \times 25 \mathrm{~mm}$ (volume $5.3 \mathrm{ml}$ ) and right ovary $27 \times 15 \times 22 \mathrm{~mm}(4.5 \mathrm{ml})$. An impression of live cervical ectopic was made (Figures 1 and 2 ). 
A second senior sonographer was called to scan, and she also agreed with the findings.

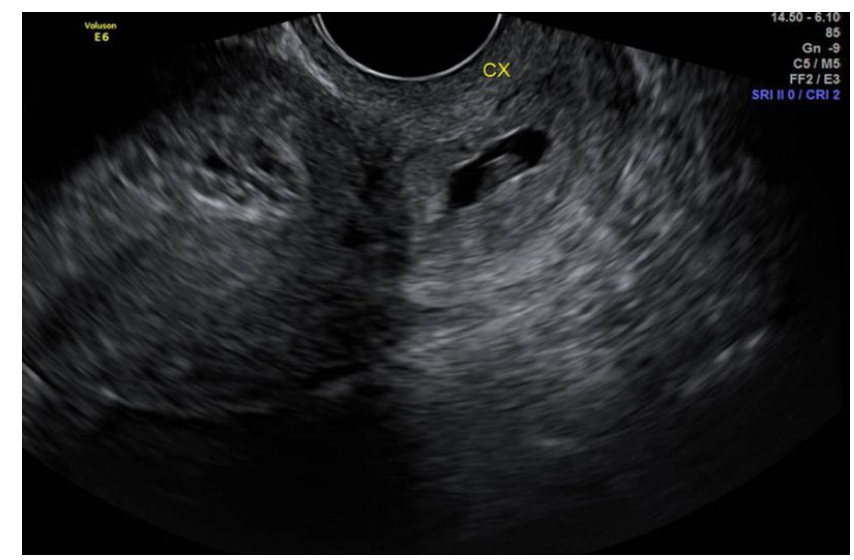

Figure 1: Transvaginal (TV) scans showing empty uterine cavity with ectopic pregnancy in the cervical canal.

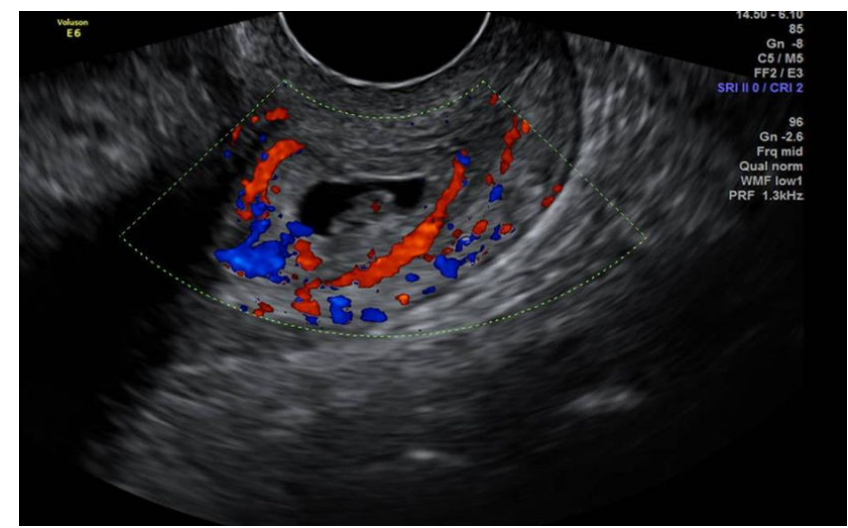

Figure 2: Vascularity of the live cervical ectopic pregnancy demonstrated on TV scan.

\section{Treatment}

Options of management were discussed with the patient including expectant, medical and surgical treatments. She consented for surgical management due to high serum B-hCG of over 10,000 U/L and presence of cardiac activity which both indicated reduced success rate for medical treatment only. Three consultant gynaecologists were present in theatre, the interventional radiologist was on standby, and the on-call haematologist and the blood transfusion laboratory were also notified in case of massive bleeding. She had hysteroscopy and suction evacuation under ultrasound guidance.

Saline hysteroscopy showed cervical ectopic embedded in the left lateral wall of the cervical canal (Figure 3). Slight dilatation and hysteroscopy showed empty uterine cavity with both ostia seen. Successful evacuation was done with size 7 cannula and products removed. Repeat hysteroscopy showed empty cervical canal and uterine cavity. Haemostatic cervical balloon (CRB) was inserted into the cervical canal and inflated with $10 \mathrm{mls}$ of fluid and vaginal balloon with $40 \mathrm{mls}$ fluid. Estimated blood loss was 80 mls with saline fluid deficit of 400 mls.
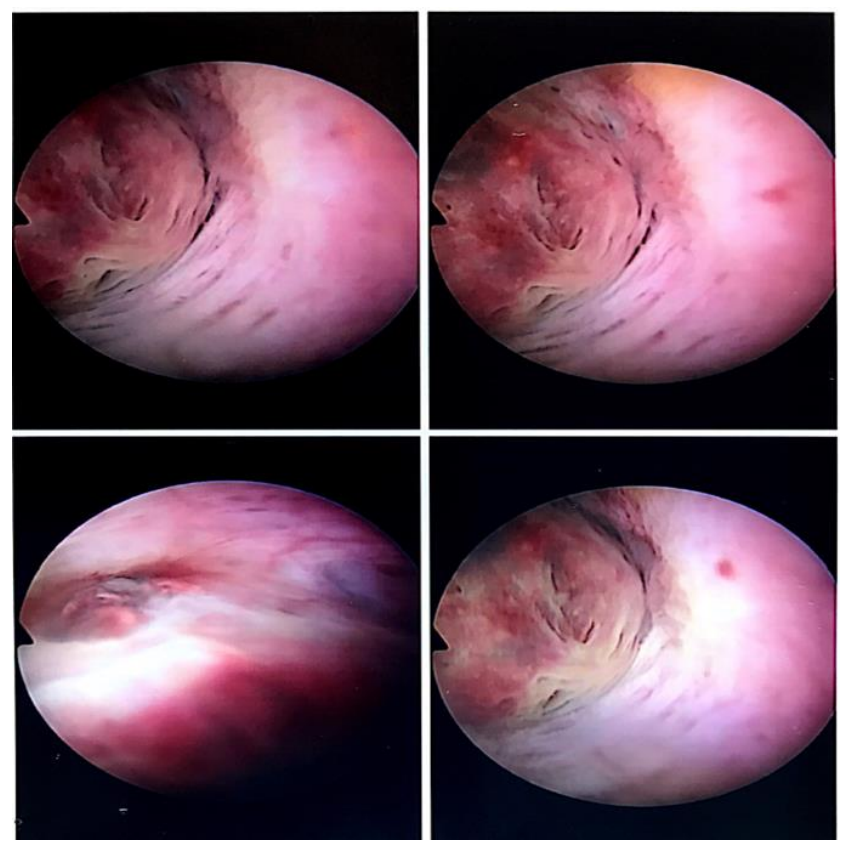

Figure 3: Hysteroscopic image showing cervical ectopic pregnancy embedded on the left lateral wall of the cervical canal.

The balloon was removed the next day with no bleeding. Patient was stable and serum B-hCG 48 hours from the first one (10713 U/L) was $4082 \mathrm{U} / \mathrm{L}$, HB level of $128 \mathrm{~g} / \mathrm{L}$ and white cell count of 10.70 cells.

She subsequently has $100 \mathrm{mg}$ of intramuscular Methotrexate (weight $90 \mathrm{~kg}$, height $156 \mathrm{~cm}$, surface area 1.97 $\mathrm{m} 2$ ) post -operatively with her consent. Levels of serum B-hCG on days 4 and 7 post methotrexate injection were $476 \mathrm{U} / \mathrm{L}$ and $145 \mathrm{U} / \mathrm{L}$ respectively. Histology showed decidua and nonmolar chorionic villi, confirming products of conception. Patient was discharged home in stable condition with contact details and information leaflets.

\section{Outcome and Follow up}

Urine pregnancy test done three weeks after the surgery was negative, and patient had no complaints. She was informed to come for early trans-vaginal ultrasound scan between 6-8 weeks once she is pregnant to confirm pregnancy location and viability. She was also advised to avoid pregnancy for the next 3 months because of the methotrexate injection

\section{Discussion and Conclusion}

CEP is a rare form of gestation with an incidence of 1 in 9000 deliveries [1]. It associated with high morbidity and potential mortality if not well managed. Historically, CEP was associated with catastrophic haemorrhage and was presumptively managed with hysterectomy [2-4]. In recent 
times, improved resolution of ultrasound and more specialised training have led to utilisation of more conservative approach with the aim of reducing bleeding and preserving reproductive potential [5].

The pathogenesis of CEP is due faulty implantation of an embryo in the endocervical canal below the internal cervical os. Possible risk factors include previous dilatation and curettage, prior caesarean section, in vitro fertilisation, chronic endometritis and uterine fibroid $[5,6]$.

An important close differential diagnosis of CEP is a miscarriage residing in the cervix, which can be easily detected by positive sliding sign. This means the gestational sac of the miscarriage slides on the cervical canal when gentle pressure is applied on the cervix with a transvaginal probe unlike in CEP [6]. Also, the presence of blood flow surrounding the gestational sac using colour Doppler technology strongly suggests CEP unlike miscarriage.

Many management options of CEP have been described in the literature including dilatation and curettage, parenteral methotrexate injection and local injection of potassium chloride or methotrexate [7]. Use of balloon catheter, uterine artery ligation and uterine artery embilisation (UAE) are important adjunctive measure used to reduce bleeding in the management of CEP [8].

Traditionally, CEP was managed with dilatation and curettage ( $D$ \& C) with some other measures to control bleeding. This is however associated with increased rate of massive bleeding needing hysterectomy [1-3]. Therefore, surgical management should be reserved for cases in which alternative options (medical treatment especially) are not suitable.

Situations associated with high failure rate of medical management with systemic methotrexate include serum BhCG above 10,000 U/L, gestational age above 9+0 weeks, crown-rump length more than $10 \mathrm{~mm}$ and presence of foetal cardiac activity $[9,10]$.

Our case demonstrated important use of MDT approach in management, and adequate preparation was made should massive intra-operative haemorrhage develop. Local and or systemic injection of methotrexate was considered but because of the high B-HCG level and presence of cardiac activity, this was not used as the primary treatment modality. The prompt use of inflated catheter balloon helped to stem massive bleeding, and the adjunctive use of post-operative methotrexate injection contributed to the treatment success.

\section{Learning points}

- CEP is a rare, accounting for less than $1 \%$ of all ectopic pregnancies.
- Accurate diagnosis of cervical ectopic requires good sonographer skills and agreed upon when possible by more than one clinician.

- Pre-operative plan requires multidisciplinary team approach in anticipation for major bleeding including interventional radiologists, haematologists, blood transfusion services, experienced gynaecologists, etc.

- An elaborate follow up plan must be put in place to ensure total resolution

\section{Acknowledgement}

- No conflicts of interest

- No funding for this clinical publication

- Patient gave consent for the publication and no revealing information of patient in keeping with ethics of publication

\section{References}

1. Samal S, Ghose S, Pallavee P (2015) Successful management of live ectopic pregnancy: A case report. J Clin Diag Res 9: 12

2. Persadie RJ, Costescu-Green D, Gerster KM (2016) Cervical ectopic pregnancy complicated by escherichia coli septicemia: A Case Report. J Obstet Gynaecol Can 38: 275-278.

3. Jurkovic D, Hacket E, Campbell S (1996) Diagnosis and treatment of early cervical pregnancy: a review and a report of two cases treated conservatively. Ultrasound Obstet Gynecol 8: 373-380.

4. Timor-Tritsch IE, Monteagudo A, Mandeville EO (1994) Successful management of viable cervical pregnancy by local injection of methotrexate guided by transvaginal ultrasonography. Am J Obstet Gynecol 170: 737-739.

5. Jessian LM, Amanda K, Katherine S (2018) Second trimester cervical ectopic pregnancy and haemorrhage: $A$ case report and review of the literature. Case Rep Obstet Gynecol 1-3.

6. Singh S (2013) Diagnosis and management of cervical ectopic pregnancy. J Hum Reprod Sci 6: 273-276.

7. Benson CB, Doubilet PM (1996) Strategies for conservative treatment of cervical ectopic pregnancy. Ultrasound Obstet Gynecol 8: 371-372.

8. Trambert JJ, Einstein MH, Banks E, Frost A (2005) Uterine artery embolization in the management of vaginal bleeding from cervical pregnancy: A case series. J Reprod Med 50: 844-850.

9. Kung FT, Chang SY (1999) Efficacy of methotrexate treatment in viable and nonviable cervical pregnancies. Am J Obstet Gynecol 181: $1438-1444$

10. Elson CJ, Salim R, Potdar N (2016) On behalf of the Royal College of Obstetricians and Gynaecologists. Diagnosis and management of ectopic pregnancy. BJOG 123: 15-55. 